\title{
A Ty1 Cell-Type-Specific Regulatory Sequence Is a Recognition Element for a Constitutive Binding Factor
}

\author{
MAHSHID COMPANY ${ }^{\dagger}$ AND BEVERLY ERREDE* \\ Department of Chemistry, University of North Carolina, Chapel Hill, North Carolina 27514
}

Received 9 June 1988/Accepted 19 September 1988

\begin{abstract}
Ty transposable-element insertion mutations of Saccharomyces cerevisiae can cause cell-type-dependent activation of adjacent-gene expression. Several cis-acting regulatory regions within Ty1 are responsible for the effect of Ty1 on adjacent-gene expression. One of these is the block II sequence that was defined by its homology to mammalian enhancers and to the yeast a1- 22 control site. Tandem copies of a 57-base-pair region encompassing block II caused an additive increase in expression of the CYC7 reporter gene in the absence of other Ty1 sequences. The activation of gene expression by the multiple repeats was abolished in a/ $\alpha$ diploid cells. A specific complex between a constitutive factor in whole-cell extracts and the DNA regulatory element was observed. The protein-binding site for the constitutive factor coincided with the block II element. Base-pair substitutions within the binding site abolished the ability of the block II element to function as a component of the Tyl activator and to form the factor-DNA complex. The correlation between complex formation and reporter gene expression indicates that factor binding to the cis-acting element is essential for this element to function as a component of the Ty1 activator.
\end{abstract}

Eucaryotic cells differentiate into distinct cell types that express characteristic subsets of genes and are able to perform specialized functions. Transcriptional regulation is one level of control for cell-type-specific gene expression. Existing evidence indicates that transcriptional regulation involves interactions between specific DNA recognition elements, site-specific DNA-binding proteins, and the transcriptional machinery (for reviews, see references 8,35 , and 45). In general, promoters of eucaryotic genes are composed of multiple DNA sequence elements which function in different combinations to achieve specific patterns of gene expression. Proteins that bind to specific DNA sequences have been identified in many systems, and some have been purified. However, the mechanisms by which these complexes control gene expression are not understood.

Saccharomyces cerevisiae is a model system for the study of events controlling cell-type-specific gene expression. There are three specialized cell types of this yeast. The a and $\alpha$ haploid cell types are specialized to mate with each other, and the $\mathbf{a} / \alpha$ diploid cell type is specialized for meiosis and sporulation (for reviews, see references 24 and 37). The genetic composition of the mating-type locus $(M A T)$ is the primary determinant of cell type. In MAT $\alpha$ cells, the $\alpha$ allele expresses two DNA-binding proteins, $\alpha 1$ and $\alpha 2$. The $\alpha 1$ protein is a positive regulator of $\alpha$-specific genes, and the $\alpha 2$ protein is a negative regulator of a-specific genes $(2,28)$. In $M A T$ a cells, the a allele expresses the al regulatory protein, which has no known function in haploid cells. In MATa/ $M A T \alpha$ diploid cells, both the a1 and $\alpha 2$ proteins are expressed. Together, a1 and $\alpha 2$ repress expression of haploidspecific genes such as MATal, STE12, Ty, and Ty-controlled genes $(9,10,16,22,31,38,50)$. Haploid-specific gene expression depends on several regulatory determinants in addition to those encoded at $M A T$. Some of these have been identified by mutations that prevent mating in both haploid cell types $(23,33)$. The STE7, STE11, and STE12 genes are

\footnotetext{
* Corresponding author.
}

† Present address: Division of Biology, California Institute of Technology, Pasadena, CA 91125. among this group, and their gene products have been shown to be required for full expression of some a-specific, $\alpha$ specific, and Ty-controlled genes $(10-12,15,50)$.

The $C Y C 7-H 2$ gene is a convenient reporter for cell-typespecific gene expression in $\mathrm{S}$. cerevisiae. The $\mathrm{CYC7}-\mathrm{H} 2$ allele was caused by insertion of a transposable element, Ty1, into the 5' noncoding region of the CYC7 gene, which codes for iso-2-cytochrome $c$. The Tyl insertion causes a 20 -fold overproduction of iso-2-cytochrome $c$ in the a and $\alpha$ haploid cell types. The overproduction is repressed in the a/ $\alpha$ diploid cell type by expression of MATa1 and MAT $\alpha 2$ (10). The overproduction is also abolished in the haploid cell types by mutations at any one of the STE7, STE11, or STE12 genes (10-12).

Mutational analysis of the CYC7-H2 Ty1 indicates that there are multiple regulatory elements with Ty1 responsible for the observed pattern of cell-type-specific gene expression. Two regions of Ty1 (Fig. 1, A and D) that are sufficient to mimic regulation of adjacent expression characteristic of the complete Ty1 were identified (6). Region A includes the U5 domain of a delta element and 145 base pairs (bp) of an adjacent epsilon sequence. This region was shown to function as a STE7- and STE12-dependent activator of CYC7 reporter gene expression (5). A DNA recognition element for a site-specific binding factor within region $\mathrm{A}$ was identified (Fig. 1, sterile responsive element [SRE]). Binding of the $S T E$-dependent protein factor was shown to be important for function of the activator sequence. A fragment from Ty1-15 that encompasses the sequence corresponding to the sterile responsive element of the $C Y C 7-H 2 \mathrm{Ty} 1$ was found to function as an activator of $P G K$ reporter gene expression (41). Region D includes the element designated block II (Fig. 1, II) which has overlapping homology to the simian virus 40 (SV40) enhancer and to the al- $\alpha 2$ repressor site found at several haploid-specific genes (13). Either region D or a synthetic block II element in the context of other Ty1 sequences functions as an orientation-independent activator of $C Y C 7$ reporter gene expression (14). Region D in the absence of other Ty1 sequences was found to cause a 2.5-fold increase of reporter gene expression in haploid cells 
A.

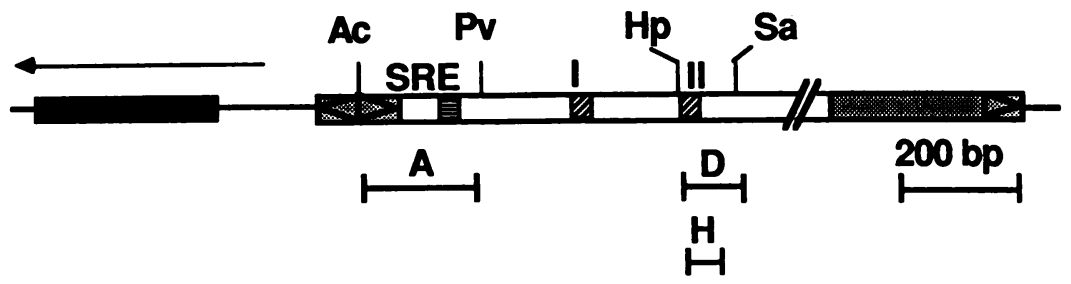

B.
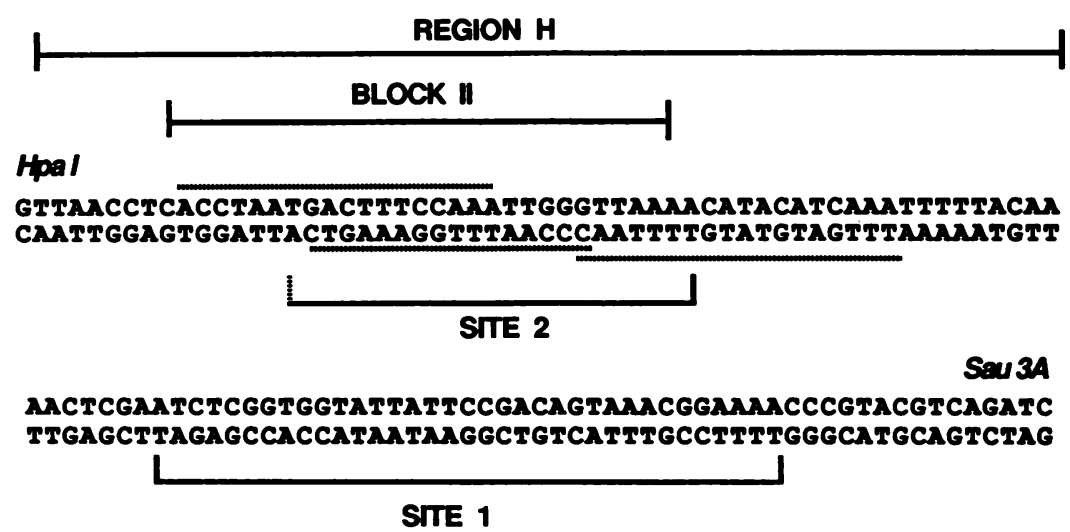

FIG. 1. (A) Location of transcriptional control regions in the CYC7-H2 Ty1. The CYC7 coding sequence is shown by the solid box. Thick lines represent $C Y C 7$ flanking sequences. The arrow above $C Y C 7$ indicates the polarity and extent of the $C Y C 7$ transcription unit. Delta sequences are shown by stippled boxes. Triangles indicate the U5 domain in delta sequences. (The 5' delta of the CYC7-H2 Ty1 is abnormal in that it consists of an inverted repeat of the U5 domain.) The open boxes represent epsilon sequences. The diagonally hatched boxes (I and II) identify block I and block II sequences. The horizontally hatched box (SRE) identifies the STE12 responsive element. Restriction sites identifying fragment-A boundaries are AccI (Ac)-PvuII (Pv), and fragment-D boundaries are HpaI (Hp)-Sau3A (Sa). (B) Nucleotide sequence of Ty1 region D (positions 665 to 779; 13). The region-H sequence (positions 666 to 722) and the block II element (positions 673 to 700 ) are indicated by bars above the sequence. The overlined sequence (positions 675 to 691) is homologous to the SV40 enhancer, and the underlined sequences (positions 682 to 696 and 696 to 712 ) are homologous to the a1- $\alpha 2$ site from MAT $\alpha$. The location of site 1 and site 2 footprints for DNA-binding factors are indicated by brackets below the sequence.

and a 5-fold repression of reporter gene expression in $\mathbf{a} / \alpha$ diploid cells $(6,14)$.

Ty1 and Ty2 are two classes of Ty elements in $S$. cerevisiae. These two classes are highly related but show divergence at the DNA sequence level (53). The Ty917 element at HIS4 is a member of the Ty 2 class. Analysis of Ty917 for cis-acting regulatory regions has shown that similar to Ty1, the Ty2 element has multiple regulatory sequences responsible for activation of gene expression $(32,42)$. Liao et al. (32) defined upstream and downstream enhancer sites with $3^{\prime}$ boarders between positions 388 to 458 and 501 to 559, respectively, of Ty917. The upstream enhancer probably corresponds to the sterile responsive element that we have defined at the $\mathrm{CYC7}-\mathrm{H} 2$ Ty1. A 55-bp sequence from Ty1 region $\mathrm{A}$ that encompasses the sterile responsive element (positions 229 to 284) shows a good match (44 of 56) to Ty917 positions 377 to 432 . However, the downstream enhancer of Ty917 does not have sequences that closely match the Ty1 block II element in region D. A sequence with homology to the SV40 enhancer in Ty917 (positions 585 to 612) was identified by Roeder et al. (42), and this region is also important for activation of adjacent-gene expression. We do not know whether either of the latter regions in the Ty2 element is functionally equivalent to the Ty1 block II element.

We have further analyzed sequences encompassing the block II element of the CYC7-H2 Ty1. We present evidence that repeats of a 57-bp region encompassing block II (Fig. 1, $\mathrm{H})$ cause an additive increase in expression of the CYC7 reporter gene in the absence of other Ty1 sequences. Activation of gene expression by the multiple repeats is abolished in $\mathrm{a} / \alpha$ diploid cells and is not dependent on the STE12 gene product in haploid cells. We also show that a sequencespecific DNA-binding protein interacts with the block II sequence. Our previous identification of a STE12-dependent regulatory element in Ty1 region $A$ together with results presented here demonstrate that at least two distinctly functioning DNA sequence elements are responsible for the pattern of cell-type-specific gene expression characteristic of Ty1.

\section{MATERIALS AND METHODS}

Chemical synthesis of DNA and cloning of synthetic DNA. Oligonucleotides were synthesized on a DNA synthesizer (model 380A; Applied Biosystems, Foster City, Calif.). The 28-bp Ty1 block II sequence was synthesized by a mutagenic procedure. Construction of the mutant bank and identification of the recombinant clones with the wild-type or mutant elements have been described elsewhere (14). The same procedures were used for construction of a 57-bp region- $\mathrm{H}$ mutant bank. Four oligonucleotides corresponding to the 
Plasmid

Schematic structure

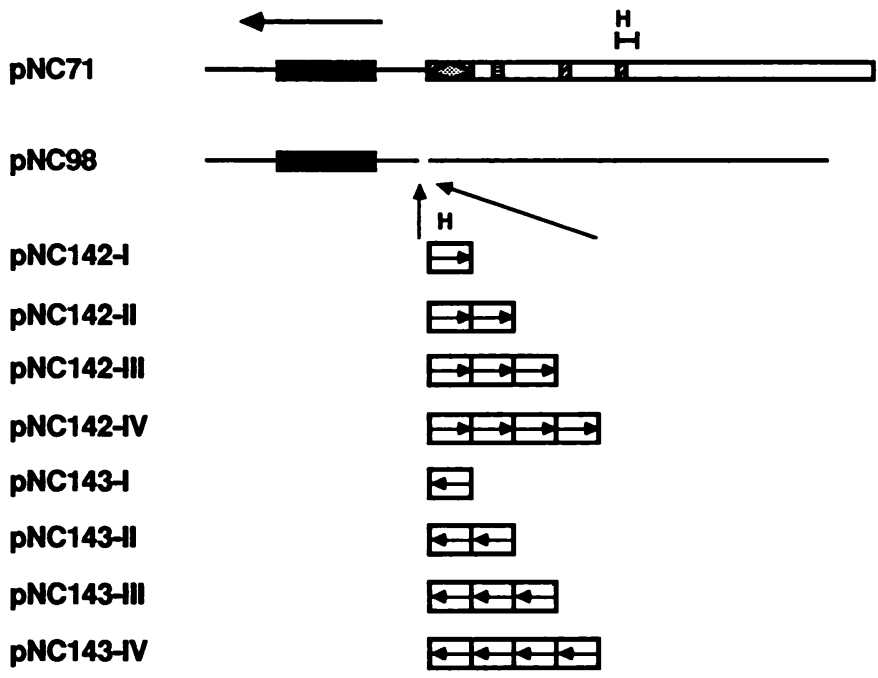

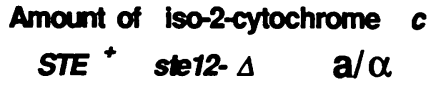

$\begin{array}{ccc}23(1) & 5(1) & 4(0.5) \\ 3(0.2) & 3(0.2) & 2(0.2) \\ 8(1) & 4(0.5) & 2(0.2) \\ 14(2) & 8(2) & 2(0.2) \\ 21(1) & 15(2) & 2(0.3) \\ 24(1) & 17(3) & 2(0.3) \\ 8(1) & 4(0.5) & 2(-) \\ 16(2) & 8(1) & 2(0.3) \\ 21(1) & 14(2) & 2(0.2) \\ 25(-) & 17(2) & 2(-)\end{array}$

FIG. 2. Structure and expression of tandem Ty1 region-H insertions at CYC7. Conventions defined in the legend to Fig. 1 are used. The Tyl fragment $\mathrm{H}$ is represented by the open box $(\Theta$, normal orientation; $\Theta$, inverted orientation). Amounts of iso-2-cytochrome $c$ are the average from determinations on 6 to 12 independent transformation isolates for each plasmid. Numbers in parentheses are standard deviations. One unit of cytochrome $c$ is defined as the amount produced by standard strains with the $C \mathrm{YC}^{+}$allele at the genomic location.

57-bp Ty1 region $\mathrm{H}$ were synthesized. Additional nucleotides to provide $S a l I$ and BamHI overhanging ends were included in the synthesis. The oligonucleotides were assembled and cloned into the SalI and BamHI sites of the M13mp11 vector. The wild-type sequence was identified among isolates from the mutant bank. Two oligonucleotides corresponding to each strand of the 28-bp MAT $\alpha$ a1- $\alpha 2$ site as defined by Siliciano and Tatchell (48) were synthesized.

Plasmids. The plasmid series designated pNC142/3-I, -II, -III, and -IV contain one, two, three, and four copies of Ty1 region $\mathrm{H}$ inserted upstream from the $C Y C 7$ reporter gene in plasmid pNC98 (Fig. 2). Plasmid pNC98, which has been described previously, is maintained in yeast cells as a low-copy-plasmid because of the presence of a centromere sequence (6). The region- $\mathrm{H}$ fragment was isolated from a M13mp11 recombinant DNA clone as a HincII-SmaI fragment. The blunt-ended fragment was cloned into the $H p a I$ site of the pNC98 polylinker. Only single insertions in each orientation were recovered, and they were designated pNC139 and pNC140. The 69-bp BglII-BamHI fragment encompassing Ty1 region $\mathrm{H}$ from $\mathrm{pNC} 139$ was isolated and cloned into the $\mathrm{Bg} I \mathrm{II}$ site of pNC98. Single and multiple insertions of region $\mathrm{H}$ were recovered in this cloning. The number of repeats and the orientation of the inserts were determined by dideoxy sequence analysis of independent clones $(1,52)$.

The $C Y C 7-H 2$ control plasmid pNC71 has been described previously (6). Plasmid pNC84 is similar to the previously described $C Y C 7-H 2$ control plasmid, pNC42 (14). In pNC84, a BamHI site was introduced at position 1259 of the $C Y C 7$ $H 2 \mathrm{Ty} 1$, whereas in pNC42 the BamHI site is at position 1068. Plasmid pNC83 is similar to the previously described pNC46 vector used for analysis of block II enhancer activity (14). In pNC83, the Ty1 sequences between the SalI and BamHI sites in pNC84 were replaced with the pNC46 polylinker. Therefore, $943 \mathrm{bp}$ of Ty1 is deleted in pNC83, compared with the 744-bp deletion in pNC46. The block II
SalI-BamHI fragments from M13mp11-T28, M13mp11-T10, and M13mp11-T74 isolates of the mutant bank (see above) were transferred to pNC83 to give pNC83-T28, pNC83-T10, and pNC83-T74, respectively.

The following plasmids were constructed for amplification of fragments used as probes or competitor DNA. Ty1 region D (HpaI-Sau3A) was isolated as a 119-bp HincII fragment from a previously described M13mp8 recombinant clone (Fig. 2 of reference 14). pNC121 contains the 119-bp HincII Tyl fragment inserted at the HincII site of pUC118 (52). The al- $\alpha 2$ site 3 from $H O$ (positions -436 to -395) was isolated from YCp50::HO as a 40-bp Sau3A-FnuDII fragment (44). pNC141 contains the 40-bp $H O$ fragment inserted into the HincII site of pUC118. pNC156 contains the 211-bp AccI$P v u I I$ Ty1 fragment (Fig. 1, region A) inserted at the HincII site of pUC118. An SphI-PvuII fragment from pSV2.CAT (nucleotide positions 253 to 325 ) that includes positions 1 to 51 of the SV40 72-bp enhancer and $22 \mathrm{bp}$ of the adjacent SV40 late leader sequence was isolated (21). pNC158 contains this 73-bp SV40 fragment inserted ai the SphI-HincII site of pUC118.

The following plasmids were used for STE7 or STE12 gene disruption. Plasmid pNC113 carries the ste7- $\Delta 3:: L E U 2$ allele. pNC113 was constructed by the same steps as described for $\mathrm{pNC} 149$ (5). $\mathrm{pNC} 113$ differs from pNC149 in that the 2.3-kb XhoI-SalI fragment from YEp13 was inserted at the HindIII deletion junction in STE7 after end repair (3). Plasmid pNC163, which carries the ste 12- $\Delta 1:: U R A 3$ allele, has been described elsewhere (5).

All plasmid constructions were made according to standard recombinant DNA procedures (35). Enzymes were purchased from New England BioLabs, Inc. (Beverly, Mass.), Bethesda Research Laboratories, Inc. (Gaithersburg, Md.), or U.S. Biochemicals (Cleveland, Ohio). All plasmids were amplified by transformation into Escherichia coli JM109 (36, 54). Plasmid DNA for isolation of probe or competitor DNAs was prepared by the method of Norgard 
TABLE 1. S. cerevisiae strains

\begin{tabular}{|c|c|}
\hline Strain & Genotype \\
\hline E906-8B. & .MATa cycl-11 cyc7-67 canl leu2-3,112 trpl- $\Delta 1$ ura3-52 \\
\hline E906-8B-8 & 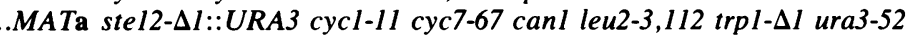 \\
\hline E730-4A.. &.$M A T \alpha$ cycl cyc7-67 canl his5 leu2 trpl \\
\hline E929-6C. & 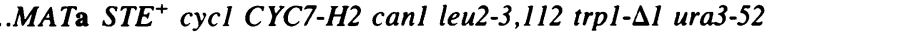 \\
\hline E929-6C-1 & 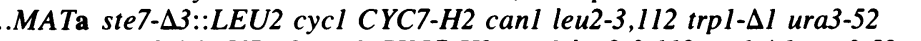 \\
\hline E929-6C-7 & .MATa ste12- $\triangle 1:: U R A 3$ cycl $C Y C 7-H 2$ canl leu2-3,112 trpl- $\Delta 1$ ura3-52 \\
\hline E929-6C-8 & .MATa $S T E^{+}$cycl CYC7-H2 canl LEU2 trpl- $\Delta 1$ ura3-52 \\
\hline \multirow[t]{2}{*}{ E93 } & 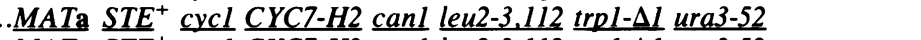 \\
\hline & $M A T \alpha S T E^{+}$cycl CYC7-H2 canl leu2-3,112 trpl- $\Delta 1$ ura3-52 \\
\hline \multirow[t]{2}{*}{ E934-1 } & 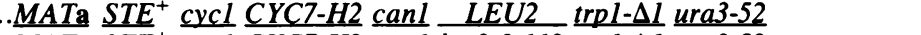 \\
\hline & 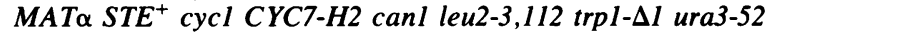 \\
\hline E934-1A. & 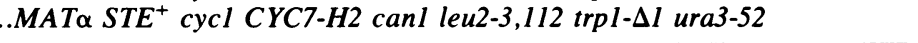 \\
\hline
\end{tabular}

(39). DNA fragments were isolated by the method of Dretzen et al. (7). Plasmid DNA for yeast transformation was prepared according to the method of Holmes and Quigley (25). Single-strand template DNA from phagemids was prepared with helper bacteriophage M13K07 according to procedures described by Vieira and Messing (52).

$S$. cerevisiae strains and genetic procedures. Complete genotypes for $S$. cerevisiae strains used in this study are given in Table 1. Strains E906-8B and E730-4A have been described previously (14). An isogenic ste 12- $\triangle 1:: U R A 3$ derivative of $\mathrm{E} 906-8 \mathrm{~B}$ and isogenic ste7- $\triangle 3:: L E U 2$, ste12$\triangle 1:: U R A 3$, and $L E U 2^{+}$derivatives of E929-6C were constructed by gene replacement (43). Strain E929-6C-1 (ste7$\triangle 3:: L E U 2)$ was constructed with the 4.4-kb SacI fragment from pNC113. Strains E929-6C-7 (ste12- $1:: U R A 3)$ and E906-8B-8 (ste12- $\Delta 1:: U R A 3)$ were constructed with the 5.5kb ClaI fragment from pNC163. Strain E929-6C-8 (LEU2 $\left.{ }^{+}\right)$ was constructed with the 2.3-kb XhoI-Sall fragment from YEp13 (3). Diploid strain E934 was constructed from E929$6 \mathrm{C}$ by use of a plasmid-borne copy of the $\mathrm{HO}$ gene carried on YCp50::HO (44). To verify genetic composition at $M A T$ and other loci, E934 was cured of the $H O$ plasmid, sporulated, and subjected to standard pedigree analysis. Strain E934-1A is a MATa segregant from sporulation of $\mathrm{E} 934 . \mathrm{A} \mathrm{LiCl}$ procedure was used for all yeast transformations (27).

Iso-2-cytochrome $\boldsymbol{c}$ determinations. Cells were grown as a narrow line under derepressing conditions on solid medium consisting of $1 \%(\mathrm{wt} / \mathrm{vol})$ yeast extract, $2 \%(\mathrm{wt} / \mathrm{vol})$ peptone, $1 \%(\mathrm{wt} / \mathrm{vol})$ sucrose, and $2 \%(\mathrm{wt} / \mathrm{vol})$ agar. Intact cells were examined at $-196^{\circ} \mathrm{C}$ for cytochrome $c$ content with a wavelength prism spectroscope (46).

Preparation of yeast cell extracts. The isogenic strains E929-6C-1, E929-6C-7, E929-6C-8, E934-1, and E934-1A were used for preparation of extracts according to previously described procedures (5). Cultures were grown in either complete medium consisting of $1 \%$ (wt/vol) yeast extract, $2 \%(\mathrm{wt} / \mathrm{vol})$ peptone, and $2 \%(\mathrm{wt} / \mathrm{vol})$ glucose or synthetic medium consisting of $0.67 \%(\mathrm{wt} / \mathrm{vol})$ yeast nitrogen base without amino acids and $2 \%$ (wt/vol) glucose supplemented with $20 \mu \mathrm{g}$ of tryptophan, $30 \mu \mathrm{g}$ of leucine, and $20 \mu \mathrm{g}$ of uracil per ml. Protein extracts were stored at $-70^{\circ} \mathrm{C}$. Protein concentrations were determined by using the protein assay kit of Bio-Rad Laboratories (Richmond, Calif.).

Probe and competitor DNA. The radiolabeled probe in these studies was Ty1 fragment D (Fig. 1). Fragment D was prepared from pNC121 as an XbaI-SalI fragment. The fragment was labeled at one or the other end with $\left[\gamma-{ }^{32} \mathrm{P}\right] \mathrm{ATP}$ (Dupont, NEN Research Products, Boston, Mass.) and polynucleotide kinase (New England BioLabs) according to standard methods (34). Typical specific activities ranged from 20,000 to $70,000 \mathrm{cpm} / \mathrm{ng}$ of DNA. Unlabeled DNA probes used for DNA-binding competition studies were isolated DNA fragments. These included the Ty1 region-D fragment from pNC121, the Ty1 block II fragments from pNC83-T28, pNC83-T10, and pNC83-T74, the Ty1 region-A fragment from pNC156, the $H O$ al- $\alpha 2$ fragment from pNC141, and the SV40 enhancer fragment from pNC158. The MAT $\alpha 1$ a1- $\alpha 2$ site used in competition assays was the appropriate picomole amount of the annealed synthetic oligonucleotides.

Gel electrophoresis, DNA binding, DNase I protection, and methylation interference assays. Protein-DNA binding reactions and electrophoretic fractionation of complexes were carried out as described by Pfeifer et al. (40) with modifications as described by Company et al. (5). Conditions for competition assays were exactly the same as those used for binding assays except that competitor DNAs were added to the reaction mix before addition of the protein extract. Binding reactions for DNase I protection assays and methylation interference assays were scaled up fivefold. The procedures used for these assays were as previously described (5).

\section{RESULTS}

Multiple tandem copies of region $H$ function as a cell-typedependent activator of gene expression. Previous analysis of the Ty1 fragment D showed that it activated reporter gene expression in the context of other Ty1 sequences $(6,14)$. Several studies have shown that some small DNA regulatory sequences can cause a manyfold activation of reporter gene expression when they are amplified $(20,49,51)$. These findings prompted us to examine the effect that amplification of the Ty1 regulatory element would have on activation of adjacent-gene expression. One to four tandem copies of a synthetic oligonucleotide corresponding to the 57-bp Ty1 region $\mathrm{H}$ were inserted in either orientation upstream of the CYC7 reporter gene in plasmid pNC98. Ty1 region $\mathrm{H}$ includes the sequences within fragment $\mathrm{D}$ defined as block II and an additional a1- $\alpha 2$ homology site adjacent to block II (Fig. 1; 13). The series of region $\mathrm{H}$ containing plasmids (pNC142-I, -II, -III, and -IV and pNC143-I, -II, -III, and -IV), the $C Y C 7$ plasmid (pNC98), and the $C Y C 7-H 2$ plasmid (pNC71) were used to transform the cytochrome $c$-deficient strain E906-8B. Amounts of iso-2-cytochrome $c$ produced by transformed strains were compared by spectroscopic examination (Fig. 2, $S T E^{+}$). The activation effect of region $\mathrm{H}$ in either orientation was approximately additive from one to three copies (pNC142/3-I, -II, and -III). Each copy caused production of an amount of iso-2-cytochrome $c$ that was 

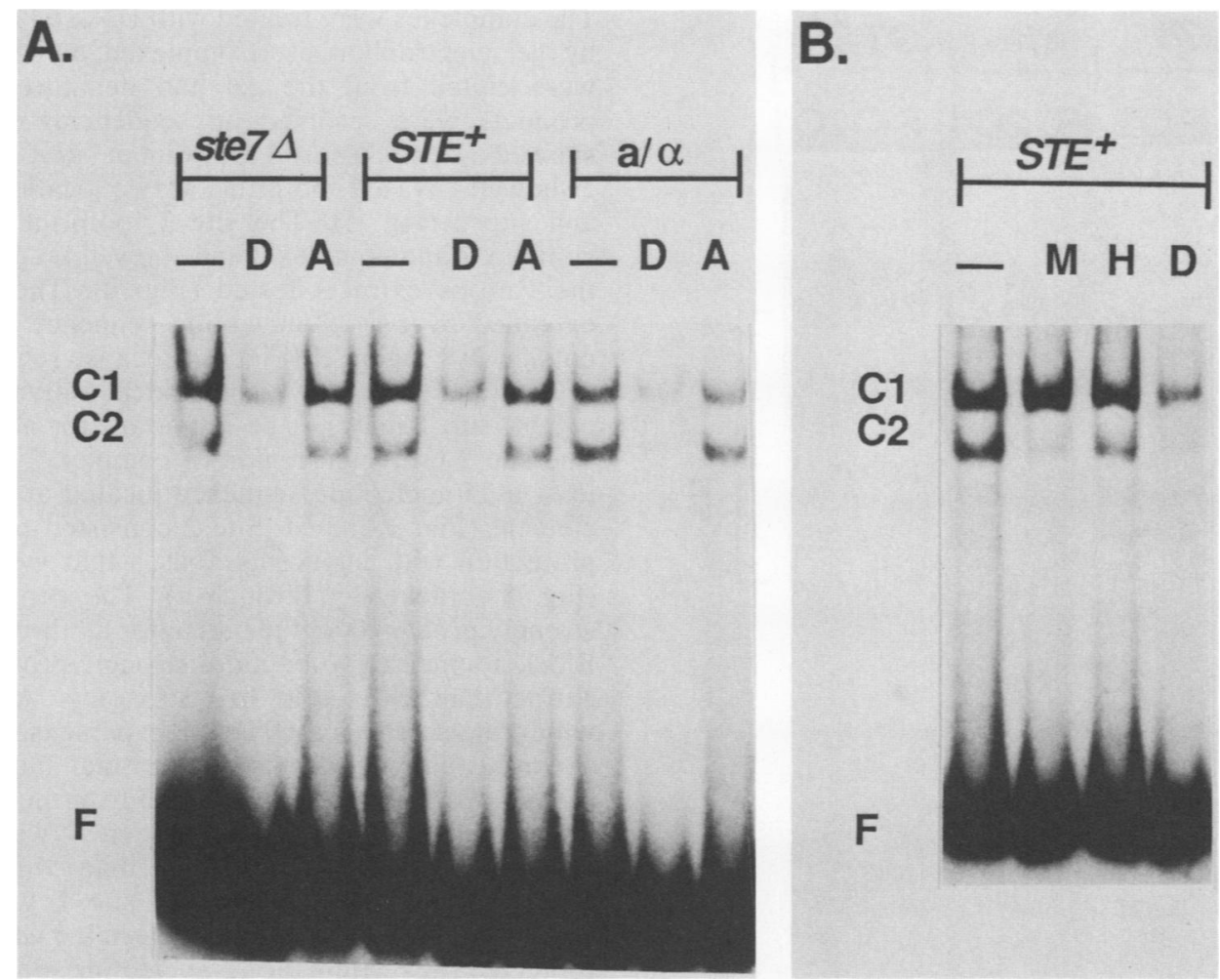

FIG. 3. Factor binding to Ty1 fragment $D$ detected by gel mobility shift assays. Extract ( $60 \mu \mathrm{g}$ of protein) prepared from yeast cells with ste $7-\Delta, S T E^{+}$, or a/ $\alpha$ genetic background was used in the binding reaction as indicated. Probe DNA was $0.5 \mathrm{ng}$ of end-labeled fragment $\mathrm{D}$. Positions of free (F), complex $1(\mathrm{C} 1)$, and complex 2 (C2) DNA probe are indicated to the left of the autoradiograms. (A) Binding reactions were performed with no specific competitor DNA (-) or with a 400 -fold molar excess of competitor fragment $D$ (D) or competitor fragment A (A). (B) Binding reactions were performed in the absence (-) or presence of a 400 -fold molar excess of $M A T \alpha(\mathrm{M}), H O(\mathrm{H})$, or fragment D (D) DNA as competitor. The MAT $\alpha$ competitor DNA is the a1- $\alpha 2$ site at positions 1671 to 1643 (48). The $H O$ competitor DNA is the al- $\alpha 2$ site 3 at positions -436 to -395 (44). The $M A T \alpha$ and $H O$ al- $\alpha 2$ sites were shown to be sufficient to direct diploid-specific transcriptional repression $(44,48)$.

about two to threefold above the background amount produced by the CYC7 control plasmid (pNC98). The additive effect of further amplification appeared to diminish between three and four tandem copies (pNC142/3-III and pNC142/ 3-IV), giving a maximal activation of approximately eightfold above the background amount.

To test the cell-type specificity of gene activation by tandem copies of region $\mathrm{H}$, the amount of iso-2-cytochrome $c$ produced in $\mathbf{a} / \boldsymbol{\alpha}$ diploid cells was examined. Diploid cells were constructed by crossing MAT $\alpha$ tester strain E730-4A to representative $M A T$ a transformed strains. Amounts of iso2-cytochrome $c$ were determined as before. In diploid cells, activation of $C Y C 7$ reporter gene expression by the one to four tandem elements (pNC142/3-I, -II, -III, and -IV) was reduced to the basal $C Y C 7$ amount (pNC98) (Fig. 2, a/ $\alpha$ ). We conclude that region $\mathrm{H}$ functions as a cell-type-specific activator of adjacent-gene expression.

Ty1 activation of $C Y C 7$ expression is known to be $S T E 7$, STE11, and STE12 dependent (10-12). Expression of STE7 and $S T E 11$ is not cell type dependent, but expression of $S T E 12$ is repressed 5 - to 10 -fold in the a/ $\alpha$ cell type $(4,16)$. Therefore, repression of the region- $\mathrm{H}$ activator observed in a/ $\alpha$ cells could be an indirect effect caused by reduced $S T E 12$ expression. To test this possibility, each of the plasmids represented in Fig. 2 was used to transform the ste 12- $\Delta 1$ strain E906-8B-8. Amounts of iso-2-cytochrome $c$ produced by transformed strains were determined as before. Activation of $C Y C 7$ expression by the tandem copies of region $\mathrm{H}$ was observed even in the complete absence of the STE12 gene product (Fig. 2, ste $12-\Delta$ ). As in the STE12 ${ }^{+}$haploid strain, the amount of activation increased as the number of tandem region- $\mathrm{H}$ copies increased. Even though the amount of activation was less in the ste 12- $\Delta l$ strain than in the $S T E 12^{+}$strain (pNC142/3-I, -II, -III, and -IV), these results exclude the possibility that the amount of repression observed in $\mathrm{a} / \alpha$ cells is caused solely by reduced STE12 expression.

Block II binds specific cellular factors. To detect proteins in yeast whole-cell extracts that bind to Ty1 sequences encompassing block II, we used a gel mobility shift assay $(17,19)$. The Ty1 fragment D (Fig. 1) isolated from pNC121 was $5^{\prime}$-end-labeled with ${ }^{32} \mathrm{P}$ and used as a probe. Binding activities from different cell types and genetic backgrounds were compared by preparing extracts from isogenic MATa ste7- $\Delta$, $M A T a S T E^{+}$, and $M A T \mathrm{a} / M A T \alpha$ strains. Each cell extract contained identical binding activities that resulted in two major bands with altered electrophoretic mobility $(\mathrm{C} 1$ and $\mathrm{C} 2$ in Fig. 3A, lanes -). Identical complexes were also observed with extracts from isogenic MAT $\alpha$ and MATa ste12- $\Delta$ strains (results not shown). A competition analysis was performed to examine the specificity of complexes formed with fragment $D$ as the probe. Addition of a 400 -fold molar excess of unlabeled fragment $D$ completely inhibited formation of complex 2 and partially inhibited formation of complex 1 (Fig. 3A, lanes D). The unrelated Ty1 region-A fragment (Fig. 1) failed to compete for the formation of either 


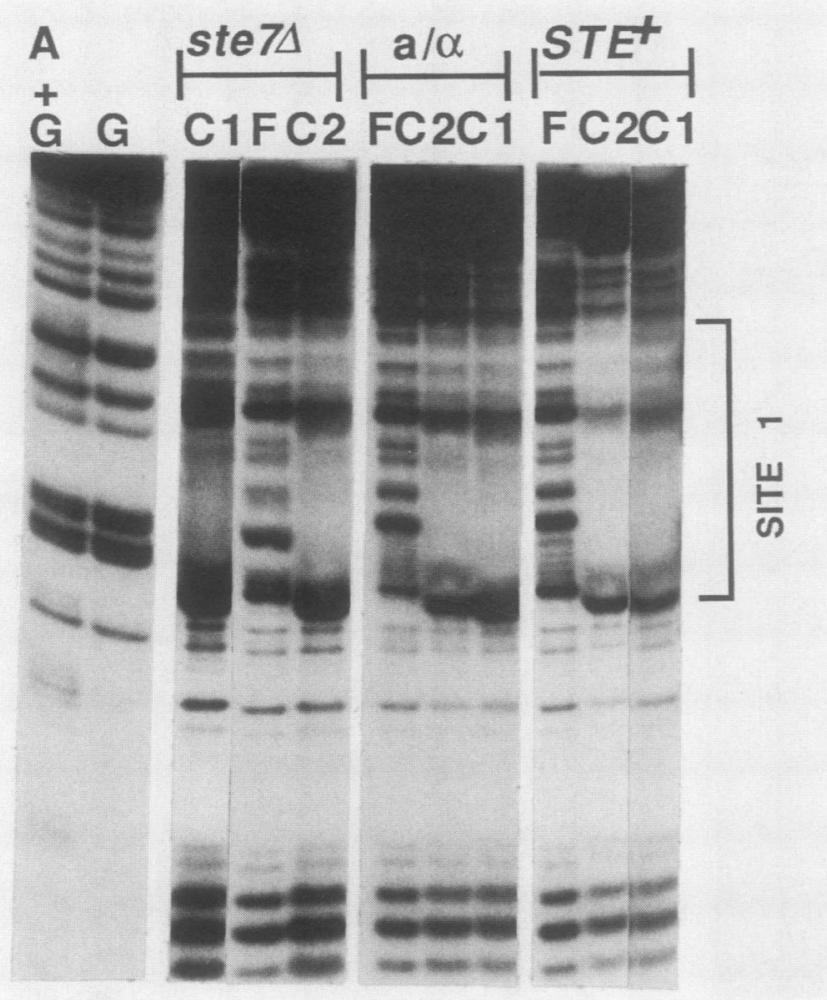

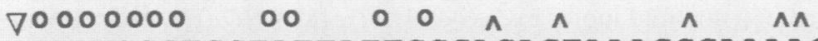
AATCTCGGTGGTATTATTCCGACAGTAAACGGAAAAC TTAGAGCCACCATAATAAGGCTGTCATTTGCСTTTTG

\section{SITE 1}

FIG. 4. DNase I protection analysis of factor-binding site 1 . The top strand of fragment D (Fig. 1) was specifically end labeled and used as probe DNA. Extracts used for factor binding were prepared

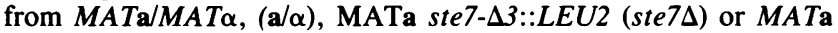
$S T E^{+}\left(S T E^{+}\right)$genetic background as indicated. Lanes display cleavage products from Maxam and Gilbert purine-specific $(A+G)$ or guanine-specific $(G)$ reactions and free $(F)$, complex $1(\mathrm{C} 1)$, and complex $2(\mathrm{C} 2)$ probe DNA. Only the portion of the autoradiogram that displays nucleotide positions 705 to 779 (13) of fragment D is shown. DNase I protected and hypersensitive sites are within the region indicated by the bracket. The nucleotide sequence of this region is given below the autoradiogram. Locations of strong protected sites $(0)$, weak protected sites $(\wedge)$, and the strongest hypersensitive sites $(\nabla)$ are indicated.

complex 1 or complex 2 (Fig. 3A, lanes A). These results showed that complexes 1 and 2 are formed by specific DNA-protein binding interactions. Although Ty1 region D encompasses a cell-type-dependent activator of gene expression, the specific binding interactions detected by the gel mobility shift assay were not cell type dependent.

Identification of protein-binding sites. A DNase I protection assay was used to map the factor-binding site(s) in $\mathrm{C} 1$ and C2 (18). The assay identifies sequences rendered inaccessible to cleavage by DNase I because of a factor bound at that site. An end-labeled fragment-D probe was prepared and incubated with extracts from $M A T \mathbf{a} / M A T \alpha$, MATa ste7- $\Delta$, and MATa $S T E^{+}$strains to allow complex formation.
The complexes were treated with DNase I and then resolved by gel electrophoresis. Complexed and free DNA bands were eluted from the gel and denatured. The resulting products were resolved on sequencing gels. Complex 1 showed a single DNase I footprint at site 1 (Fig. 4). Complex 2 showed DNase I footprints at two locations, site 1 (Fig. 4) and site 2 (Fig. 5). The site 1 footprint was identical in complex 1 and complex 2 and showed no differences among the various extracts tested (Fig. 4). The site 1 footprint extended over a 35-nucleotide sequence that was located outside Ty1 region $\mathrm{H}$ (Fig. 1). Because region $\mathrm{H}$ was shown to function as a cell-type-dependent activator upon amplification, site 1 may not be important for activator function. The site 2 protected region of complex 2 extended over at least a 25 -nucleotide sequence located at the Ty1 block II element (Fig. 1 and 5). Site 2 consisted of three blocks of protection with intervening bases that were not protected (Fig. 5, brackets a through c). The central block b was strongly protected and identical for all three extracts tested. Block a appeared to be more strongly protected with $S T E^{+}$ extract than with $a / \alpha$ or ste7- $\Delta$ extracts. Another difference within block a was a DNase I hypersensitive site that was apparent with $\mathrm{a} / \alpha$ and $s t e 7-\Delta$ extract but not with $S T E^{+}$ extract. Block c was more weakly protected with all extracts; however, the protected sites were more readily discernible with $S T E^{+}$extract than with $\mathrm{a} / \alpha$ or ste $7-\Delta$ extracts. The left boundary of site 2 was not precisely defined because we could not determine whether there were protected sites within the 12-nucleotide segment at the $5^{\prime}$ end of fragment $D$.

A methylation interference assay was also used to map factor-binding sites in complex 1 and complex 2 (47). The methylation interference assay identifies guanine residues that are required for base-specific contacts with a binding factor. Methylation of guanine at the N-7 position interferes with critical contacts made by proteins in the major groove of the DNA helix. In this assay, the end-labeled fragment D was partially methylated with dimethylsulfate before complex formation. Complexes were allowed to incubate with the indicated cell extract and then resolved by gel electrophoresis. Free and complexed bands were eluted from the gel and cleaved with piperidine. Resulting products were fractionated on sequencing gels. As with the DNase I footprints, the methylation interference sites were identical for extracts from different cell types and genetic backgrounds (Fig. 6). No major-groove contacts were detected by this assay for complex 1 . Two guanine residues on each strand that are essential for base-specific contacts with the binding factor were detected in complex 2 . The four guanine residues were located within the site 2 DNase I footprint at block II (Fig. 5).

Factor binding to the block II sequence is important for gene activation observed in vivo. The results from binding and footprint assays showed that the block II sequence encompasses a recognition element for a site-specific binding factor. The 28-bp block II sequence was previously shown to function as one component of the Ty1 activator (14). A reasonable prediction is that the DNA-protein interaction at block II is important for the function of block II as a component of the Tyl activator. A test of this prediction is to compare the normal and base-pair substitution DNA elements for ability to activate gene expression in vivo and to bind specific protein factors in vitro. A single copy of the 28-bp block II fragment provides the function of a 943-bp fragment of Ty1 required for activation of the CYC7 reporter gene (Fig. 7, pNC83-T28). Two single base-pair mutations of 

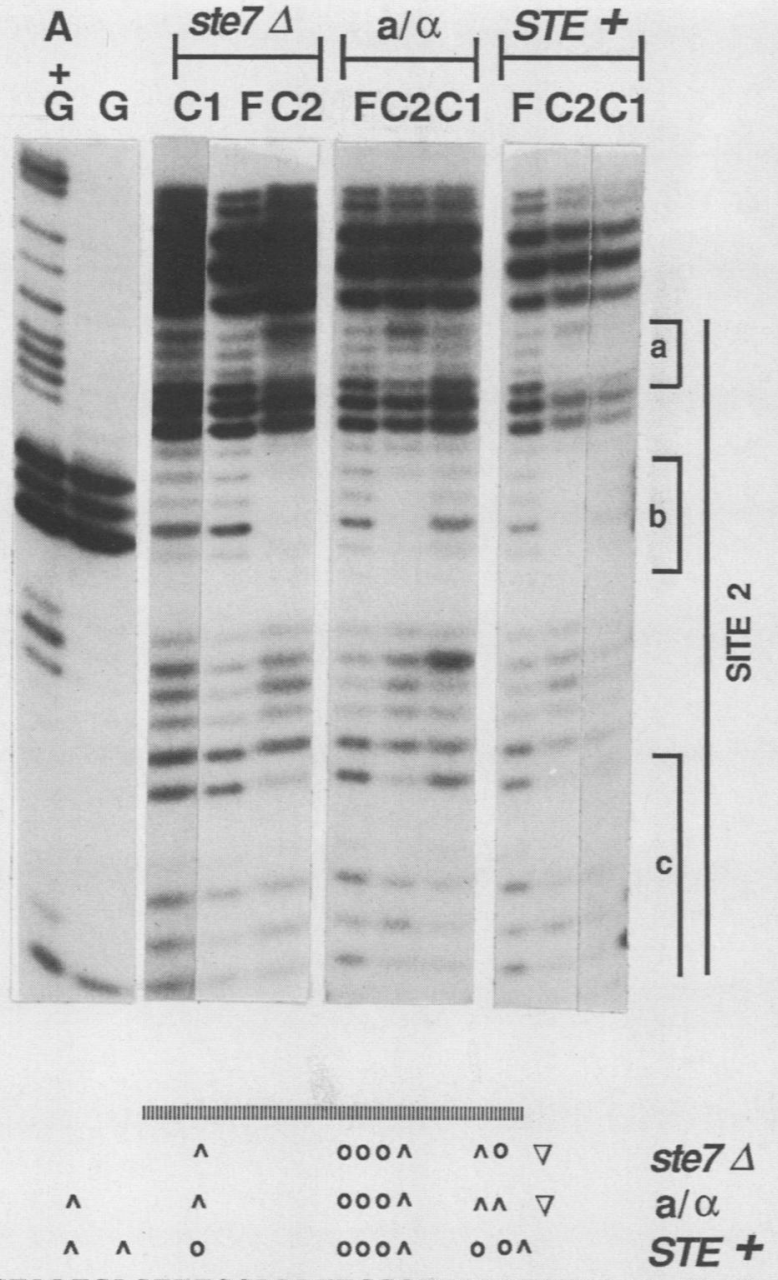

CACCTAATGACTTTCCAAATTGGGTTAAAACATACATCAAA GTGGATTACTGAAAGTTTAACCCAATTTGTATGTAGTTT

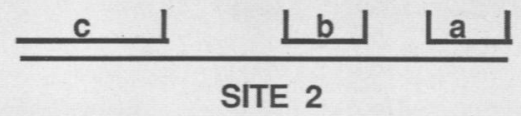

FIG. 5. DNase I protection analysis of factor-binding site 2 . Probe DNA and lanes of the autoradiogram are as defined in the legend to Fig. 4. Only the portion of the autoradiogram that displays nucleotide positions 681 to 713 (13) of fragment $D$ is shown. DNase I protected and hypersensitive sites are within regions indicated by brackets $a, b$, and $c$. The nucleotide sequence encompassing these regions is given below the autoradiogram. Locations of strong protected sites $(0)$, weak protected sites $(\wedge)$, and the strongest hypersensitive sites $(\nabla)$ corresponding to each extract are indicated. The broken horizontal bar indicates the fragment-D sequence with a 12-of-16 match to the P-site consensus sequence (2). Methylation interference sites defined in Fig. 6 are the underlined $G$ residues in the sequence.

the block II element failed to function as a component of the Ty1 activator (Fig. 7, pNC83-T10 and pNC83-T74). The normal block II element and the two substitution derivatives of the element were tested for ability to inhibit formation of complex 2 with Ty1 fragment $D$ as the radioactive probe. The normal block II element was as efficient a competitor for formation of complex 2 as was the unlabeled fragment $D$ (Fig. 8, T28 and D). Formation of complex 1, which involves a protein-binding site outside the block II sequence, was not inhibited by addition of the block II element. The substitution derivatives that were nonfunctional as components of the Ty1 activator (T10 and T74) were about eight times less efficient as competitors for formation of complex 2 than was the normal element (T28). (Compare Fig. 8, lanes T10-800 and T74-800 with lane T28-100.) The direct correlation between the ability of a specific DNA sequence to function as a component of the Ty1 activator and to bind protein factors indicates that specific protein binding at the block II DNA recognition element is crucial for the function of this element.

Other haploid-specific genes have DNA sequence elements that interact with the Ty block II binding factor. The factorbinding site 2 coincides with the SV40 enhancer core sequence and the a1- $\alpha 2$ site at block II. A competition analysis was performed to evaluate the significance of either of these homologies for binding of the factor at site 2. A 400 -fold molar excess of an unlabeled SV40 enhancer DNA fragment did not compete with Ty1 fragment $D$ for formation of either complex 1 or complex 2 (data not shown). This result indicated that the SV40 homology element of block II is not sufficient for binding of the factor. Fragments encompassing functional a1- $\alpha 2$ sites from $M A T \alpha$ and $H O$ were used in competition assays to test the importance of the block II a1- $\alpha 2$ homology in factor recognition $(44,48)$. A 400 -fold molar excess of each competed with radiolabeled fragment $D$ for complex 2 formation but had no effect on complex 1 formation (Fig. 3B, lanes $M$ and $H$ ). These data suggest that the al- $\alpha 2$ region of block $\mathrm{II}$ is the more important component of the binding-factor recognition element. They further suggest the possibility that the block II sequence binding factor may recognize and bind more or less effectively to a1- $\alpha 2$ sites present in the regulatory regions of other haploid specific genes.

\section{DISCUSSION}

Ty1 region-H activator sequence. In several cases, it has been shown that Ty insertion mutations activate adjacentgene expression in haploid cells but not in $\mathbf{a} / \alpha$ diploid cells $(10,50)$. The haploid-specific activation by $\mathrm{Ty}$ requires expression of the $S T E 7, S T E 11$, and STE12 gene products. Repression of Ty activation in diploid cells requires expression of the MATa1 and MAT 2 gene products. Two regions of Ty1 have been identified as important for the STE and a/ $\alpha$ regulation characteristic of Ty1. Region A (Fig. 1) includes a STE7- and STE12-dependent activator of reporter gene expression (5). Region D (Fig. 1) functions as an enhancer in the context of other Ty1 sequences. Region D by itself or in association with region $A$ causes repression of gene expression in diploid cells $(6,14)$. The regulatory role of a subfragment from region $D$ (Fig. 1, fragment $H$ ) which includes the block II sequence and an adjacent a1- $\alpha 2$ site was investigated. Tandem repeats of Ty1 fragment $\mathrm{H}$ were found to cause additive increases in expression of the $C Y C 7$ reporter gene in the absence of other Tyl sequences. We assume that sufficient regulatory information is included in fragment $\mathrm{H}$ and that when this information was amplified, up to an eightfold activation of $C Y C 7$ gene expression was observed in the absence of other Ty 1 sequences. The fragment $\mathrm{H}$ dependent activation was observed in haploid cell types with $S T E^{+}$or ste 12- $\Delta$ genetic background but was repressed in the a/ $\alpha$ diploid cell type. These results demonstrate that fragment $\mathrm{H}$ encompasses a cell-type-specific activator element. They also exclude the possibility that cell type specificity of the activator is an indirect consequence of reduced 


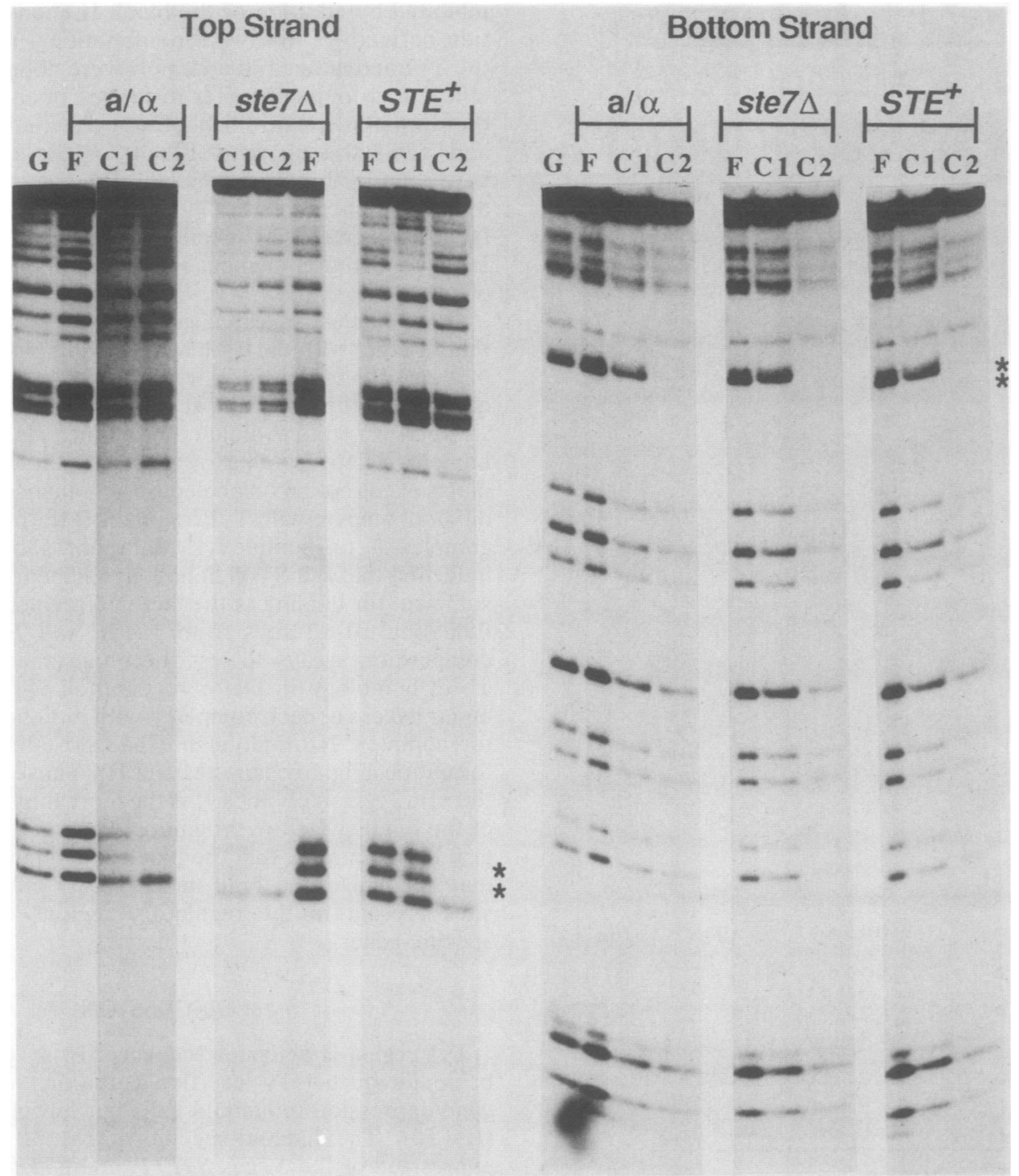

FIG. 6. Methylation interference analysis of factor-binding sites. The top or bottom strand of fragment D was specifically end labeled, treated with dimethylsulfate, and used as probe DNA. Extracts used for factor binding were prepared from yeast cells with a/ $\alpha$, ste7- $\Delta$, or $S T E^{+}$genetic background. Lanes display cleavage products from the Maxam and Gilbert guanine-specific reaction (G) and free (f), complex $1(\mathrm{C} 1)$, and complex $2(\mathrm{C} 2)$ probe DNA. *, Positions of methylation interference.

STE12 expression known to occur in the a/ $\alpha$ cell type (16). We conclude that the cell type specificity of Ty effects on adjacent-gene expression involves at least two distinctly functioning regulatory elements. One is the $S T E$-responsive element in region A and the other is the cell-type-responsive element in region $\mathrm{H}$.

Ty1 region-H protein-binding recognition element. Results presented here provide evidence that a constitutive factor binds specifically to a site located in the block II sequence of the region-H activator. The block II sequence was defined at positions 668 to 700 by its homology to the SV40 enhancer and the a1- $\alpha 2$ repressor site from MAT $\alpha 1$ (13). The factorbinding site identified by a DNase I protection assay coincides with block II (Fig. 1). The methylation interference assay identified four guanine residues, at positions 687 and 688 on the bottom strand and positions 695 and 696 on the top strand, that are points of major-groove contacts with the binding factor (Fig. 5). The two pairs of guanine residues are located on the same side of the helix. An SV40 enhancer DNA fragment did not compete with the block II sequence for factor binding. DNA fragments encompassing functional a1- $\alpha 2$ sites from two haploid-specific genes, MAT $\alpha$ and $H O$, did compete with the Ty1 block II fragment for factor binding at this site. These results indicate that the a1- $\alpha 2$ homology but not the SV40 enhancer homology includes the critical determinants of the factor recognition element at block II.

A synthetic block II element was previously shown to function as one component of the Ty1 activator. Two single base-pair substitutions of the block II sequence were nonfunctional (Fig. 7; 14). The 28-bp block II element could compete for factor binding to the fragment-D probe. The base-pair substitution derivatives that failed to function as a component of the Ty1 activator also failed to compete for factor binding. The correlation between the ability of block II to bind the factor in vitro and the ability to function as a 
Plasmid

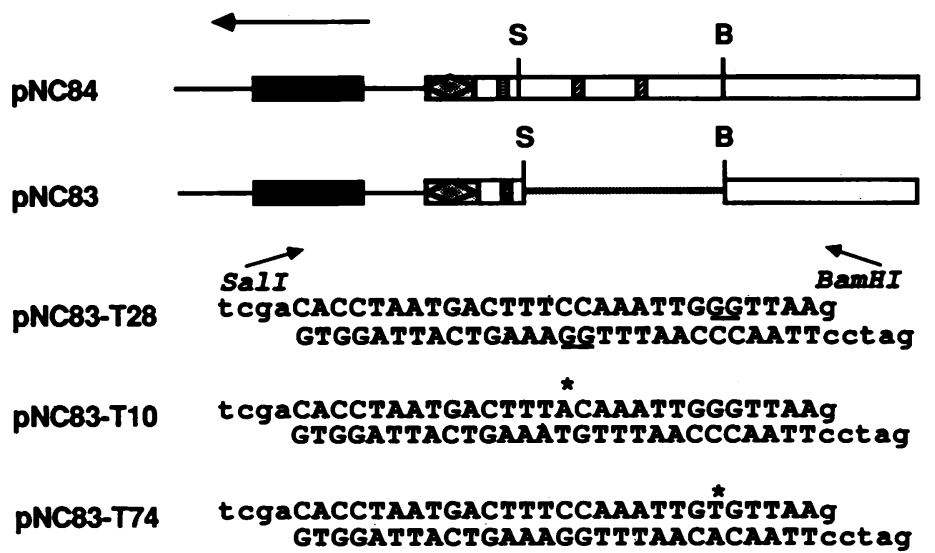

Amt of

cytochrome

25

3

FIG. 7. Structure and expression of normal and mutant block II insertions at CYC7-H2. Conventions defined in the legend to Fig. 1 are used. Linker cloning sites constructed in Tyl are Sall (S) and BamHI (B). Positions of methylation interference are underlined, and positions of base-pair substitutions in the mutant block II elements are indicated by an asterisk. Amounts of iso-2-cytochrome $c$ are the average from determinations on six independent transformation isolates for each plasmid. One unit of cytochrome $c$ is defined as the amount produced by standard strains with the $C Y C 7^{+}$allele at the genomic location.

component of the Tyl activator indicates that factor binding to the cis-acting element is essential for the function of block II as an activator of adjacent-gene expression.

Postulated activities of the constitutive block II binding factor. Activation of reporter gene expression of Tyl region $\mathrm{H}$ is repressed in diploid cells. However, the binding factor we detect is expressed in extracts prepared from both

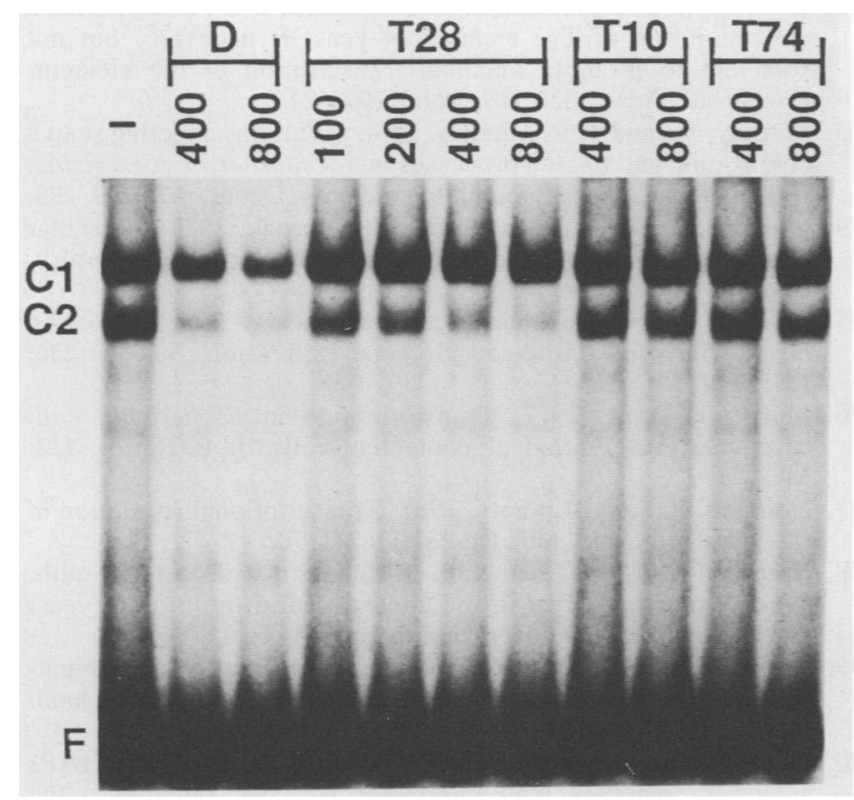

FIG. 8. Competition analysis for factor binding to normal and mutant block II DNA. Binding reactions with radiolabeled Ty1 fragment $D(0.5 \mathrm{ng})$ and extract $(60 \mu \mathrm{g}$ of protein) from yeast cells with $S T E^{+}$genetic background were performed in the absence (-) or in the presence of the specified molar excess of unlabeled competitor DNA. Competitor DNAs were fragment D (D), wildtype block II (T28), and mutant block II (T10 or T74) DNA. (See Fig. 7 for DNA sequences of the T28, T10 and T74 block II elements.) haploid and diploid cell types. Several possible models can be proposed to account for these observations. One model is that the binding factor has distinct DNA binding and activation domains similar to those of other yeast activator proteins $(26,29)$. According to this model, the binding domain is functional in all cell types. In $\mathbf{a}$ and $\alpha$ haploid cell types, the activation domain is functional and factor binding causes activation of gene expression. In the $a / \alpha$ diploid cell type, the activation domain is modified so as to be nonfunctional and factor binding causes repression of gene expression. Because the factor-binding site is homologous to the al- $\alpha 2$ repressor site, an alternative model is that the a1- $\alpha 2$ repressor in diploid cells competes with or interacts with the constitutive factor for binding. In this case, we would assume that the in vitro binding assay we use is not sufficiently sensitive to detect the a1- $\alpha 2$ binding factor produced in physiological amounts. A precedent for the latter model is provided by recent findings concerning a- and $\alpha$-specific DNA recognition elements and their specific binding factors $(2,30)$.

One candidate for the constitutive binding factor we.detect is the pheromone and receptor transcription factor (PRTF) described by Bender and Sprague (2). PRTF has been shown to bind a sequence found in the UAS region of several a- and $\alpha$-specific genes. A perfect palindrome sequence (P site, TTTCCTAATTAGGAAA) was deduced from the naturally occurring elements. A synthetic $P$ site was shown be a functional activator of gene expression and a binding site for PRTF. A comparison of Tyl block II and P-site sequences shows that the two are similar (12-of-16 match, Fig. 5). Excess unlabeled Ty1 fragment D has been found to inhibit purified PRTF from binding to a synthetic PAL DNA probe as well as to a probe containing the UAS sequence from the a-specific $S T E 2$ gene (G. Ammerer and B. Errede, unpublished observations). One possibility to consider is that PRTF plays a role in regulation of some haploid-specific genes as well as the a- and $\alpha$-specific genes. Another possibility is that there is a family of PRTF-like DNAbinding proteins which bind to related but nonidentical DNA sequences. 


\section{ACKNOWLEDGMENTS}

We thank Clyde Hutchison III for the M13mp11 recombinant carrying the 57-bp Tyl region-H fragment. We also thank Kelly Tatchell for critical reading of the manuscript.

This work was supported by Public Health Service research grant GM30619 from the National Institutes of Health.

\section{LITERATURE CITED}

1. Bankier, A. T., K. M. Weston, and B. G. Barrell. 1987. Random cloning and sequencing by the M13/dideoxynucleotide chain termination method. Methods Enzymol. 155:51-93.

2. Bender, A., and G. F. Sprague, Jr. 1987. MAT $\alpha$ l protein, a yeast transcription activator, binds synergistically with a second protein to a set of cell-type-specific genes. Cell 50:681-691.

3. Broach, J. R., J. N. Strathern, and J. B. Hicks. 1979. Transformation in yeast: development of a hybrid cloning vector and isolation of the CANI gene. Gene 8:121-133.

4. Chaleff, D. T., and K. Tatchell. 1985. Molecular cloning and characterization of the STE7 and STE11 genes of Saccharomyces cerevisiae. Mol. Cell. Biol. 5:1878-1886.

5. Company, M., C. Adler, and B. Errede. 1988. Identification of a Ty1 regulatory sequence responsive to STE7 and STE12. Mol. Cell. Biol. 8:2545-2554.

6. Company, M., and B. Errede. 1987. Cell-type-dependent activation by yeast transposon $\mathrm{Ty} 1$ involves multiple regulatory determinants. Mol. Cell. Biol. 7:3205-3211.

7. Dretzen, G., M. Bellard, P. Sassone-Corsi, and P. Chambon. 1981. A reliable method for the recovery of DNA fragments from agarose and acrylamide gels. Anal. Biochem. 112:295-298.

8. Dynan, W. S., and R. Tjian. 1985. Control of eukaryotic messenger RNA synthesis by sequence-specific DNA-binding proteins. Nature (London) 316:774-778.

9. Elder, R. T., T. P. St. John, D. T. Stinchcomb, and R. W. Davis. 1981. RNA homologous to Ty1. Cold Spring Harbor Symp. Quant. Biol. 45:581-584.

10. Errede, B., T. S. Cardillo, F. Sherman, E. Dubois, J. Deschamps, and J. M. Wiame. 1980. Mating signals control expression of mutations resulting from insertion of a transposable repetitive element adjacent to diverse yeast genes. Cell 22:427-436.

11. Errede, B., T. S. Cardillo, M. A. Teague, and F. Sherman. 1984. Identification of regulatory regions within the Ty1 transposable element that regulate iso-2-cytochrome $c$ production in the CYC7-H2 yeast mutant. Mol. Cell. Biol. 4:1393-1401.

12. Errede, B., T. S. Cardillo, G. Wever, and F. Sherman. 1981. Studies on transposable elements in yeast. I. ROAM mutations causing increased expression of yeast genes: their activation by signals directed toward conjugation functions and their formation by insertion of Ty1 repetitive elements. Cold Spring Harbor Symp. Quant. Biol. 45:593-602.

13. Errede, B., M. Company, J. D. Ferchak, C. A. Hutchison III, and W. S. Yarnell. 1985. Activation regions in a yeast transposon have homology to mating type control sequences and to mammalian enhancers. Proc. Natl. Acad. Sci. USA 82:54235427.

14. Errede, B., M. Company, and C. A. Hutchison III. 1987. Ty1 sequence with enhancer and mating-type-dependent regulatory activities. Mol. Cell. Biol. 7:258-265.

15. Fields, S., D. T. Chaleff, and G. F. Sprague, Jr. 1988. Yeast STE7, STE11, and STE12 genes are required for expression of cell-type-specific genes. Mol. Cell. Biol. 8:551-556.

16. Fields, S., and I. Herskowitz. 1987. Regulation by the yeast mating type locus of STE12, a gene required for cell-typespecific expression. Mol. Cell. Biol. 7:3818-3821.

17. Fried, M., and D. M. Crothers. 1981. Equilibria and kinetics of lac repressor-operator interactions by polyacrylamide gel electrophoresis. Nucleic Acids Res. 9:6505-6525.

18. Galas, D. J., and A. Schmitz. 1978. DNase I footprinting: a simple method for the detection of protein-DNA binding specificity. Nucleic Acids Res. 5:3157-3170.

19. Garner, M. M., and A. Revzin. 1981. A gel electrophoresis method for quantifying the binding of proteins to specific DNA regions. Application to components of the Escherichia coli lactose operon regulatory system. Nucleic Acids Res. 9:30473060.

20. Gerster, T., P. Matthias, M. Thali, J. Jiricny, and W. Schafiner. 1987. Cell-type-specificity elements of the immunoglobulin heavy chain gene enhancer. EMBO J. 6:1323-1330.

21. Gorman, C. 1986. High efficiency gene transfer into mammalian cells, p. 143-190. In D. M. Glover, R. Rickwood, and B. D. Hames (ed.), DNA cloning, vol. 2: A practical approach. IRL Press, Washington D.C.

22. Goutte, C., and A. D. Johnson. 1988. al protein alters the DNA binding specificity of $\alpha 2$ repressor. Cell 52:875-882.

23. Hartwell, L. H. 1980 . Mutants of Saccharomyces cerevisiae unresponsive to cell division control by polypeptide mating hormone. J. Cell. Biol. 85:811-822.

24. Herskowitz, I. 1986. Specialized cell types in yeast: their use in addressing problems in cell biology, p. 625-656. In J. Hicks (ed.), Yeast cell biology. Alan R. Liss, Inc., New York.

25. Holmes, D. S., and M. Quigley. 1981. A rapid boiling method for the preparation of bacterial plasmids. Anal. Biochem. 114:193197.

26. Hope, I., and K. Struhl. 1986. Functional dissection of a eukaryotic transcriptional activator protein, GCN4 of yeast. Cell 46:885-894.

27. Ito, H., Y. Fukuda, K. Murate, and A. Kimura. 1983. Transformation of intact yeast cells treated with alkali cations. J. Bacteriol. 153:163-168.

28. Johnson, A. D., and I. Herskowitz. 1985. A repressor (MATa2 product) and its operator control expression of a set of cell type specific genes in yeast. Cell 42:237-247.

29. Keegan, L., G. Gill, and M. Ptashne. 1986. Separation of DNA binding from the transcription-activating function of a eukaryotic regulatory protein. Science 231:669-704.

30. Keleher, C. A., C. Goutte, and A. D. Johnson. 1988. The yeast cell-type-specific repressor $\alpha 2$ acts cooperatively with a noncell-type-specific protein. Cell 53:927-936.

31. Klar, A. J. S., J. N. Strathern, J. R. Broach, and J. B. Hicks. 1981. Regulation of transcription in expressed and unexpressed mating type cassettes of yeast. Nature (London) 289:239-244.

32. Liao, X., J. J. Clare, and P. J. Farabaugh. 1987. The upstream activation site of Ty2 element of yeast is necessary but not sufficient to promote maximal transcription of the element. Proc. Natl. Acad. Sci. USA 84:8520-8524.

33. Mackay, V., and T. R. Manney. 1974. Mutations affecting sexual conjugation and related processes in Saccharomyces cerevisiae. II. Genetic analysis of nonmating mutants. Genetics 76:273-288.

34. Maniatis, T., E. F. Fritsch, and J. Sambrook. 1982. Molecular cloning: a laboratory manual. Cold Spring Harbor Laboratory, Cold Spring Harbor, N.Y.

35. Maniatis, T., S. Goodbourn, and J. S. Fischer. 1987. Regulation of inducible and tissue-specific gene expression. Science 236: 1237-1245.

36. Morrison, D. A. 1977. Transformation in Escherichia coli: cryogenic preservation of competent cells. J. Bacteriol. 132: 349-351.

37. Nasmyth, K., and D. Shore. 1987. Transcriptional regulation in the yeast life cycle. Science 237:1162-1170.

38. Nasmyth, K. A., K. Tatchell, B. D. Hall, C. Astell, and M. Smith. 1981. A position effect in the control of transcription at yeast mating type loci. Nature (London) 289:244-250.

39. Norgard, M. V. 1981. Rapid and simple removal of contaminating RNA from plasmid DNA without use of RNase. Anal. Biochem. 113:34-42.

40. Pfeifer, K., B. Arcangioli, and L. Guarente. 1987. Yeast HAP1 activator competes with the factor $\mathrm{RC} 2$ for binding to the upstream activation site UAS1 of the CYC1 gene. Cell 49:9-18.

41. Rathjen, P. D., A. J. Kingsman, and S. M. Kingsman. 1987. The yeast ROAM mutation-identification of the sequences mediating host gene activation and cell-type control in the yeast retrotransposon, Ty. Nucleic Acids Res. 15:7309-7324.

42. Roeder, G. S., A. B. Rose, and R. E. Pearlman. 1985. Transposable element sequences involved in the enhancement of yeast gene expression. Proc. Natl. Acad. Sci. USA 82:5428-5432.

43. Rothstein, R. 1983. One-step gene disruption in yeast. Methods 
Enzymol. 101:202-211.

44. Russell, D. W., R. Jensen, M. J. Zoller, J. Burke, B. Errede, M. Smith, and I. Herskowitz. 1986. Structure of the Saccharomyces cerevisisiae $\mathrm{HO}$ gene and analysis of its upstream regulatory region. Mol. Cell. Biol. 6:4281-4294.

45. Serfling, E., M. Jasin, and W. Schaffner. 1985. Enhancers and eukaryotic gene transcription. Trends Genet. 1:224-230.

46. Sherman, F., and P. P. Slonimski. 1964. Respiration-deficient mutants of yeast. II. Biochemistry. Biochim. Biophys. Acta 90: $1-15$.

47. Siebenlist, U., and W. Gilbert. 1980. Contacts between Escherichia coli RNA polymerase and an early promoter of the phage T7. Proc. Natl. Acad. Sci. USA 77:122-126.

48. Siliciano, P. G., and K. Tatchell. 1986. Identification of the DNA sequences controlling expression of the MAT $\alpha$ locus of yeast. Proc. Natl. Acad. Sci. USA 83:2320-2324.

49. Stuart, G. W., P. F. Searle, H. Y. Chen, R. L. Brinster, and R. D. Palmiter. 1984. A 12-base-pair DNA motif that is repeated several times in metallothinonein gene promoters confers metal regulation to a heterologous gene. Proc. Natl. Acad. Sci. USA 81:7318-7322.

50. Taguchi, A. K. W., M. Ciriacy, and E. T. Young. 1984. Carbon source dependence of transposable element-associated gene activation in Saccharomyces cerevisiae. Mol. Cell. Biol. 4:6168.

51. Veldman, G. M., S. Lupton, and R. Kamen. 1985. Polyomavirus enhancer contains multiple redundant sequence elements that activate both DNA replication and gene expression. Mol. Cell. Biol. 5:649-658.

52. Vieira, J., and J. Messing. 1987. Production of single-stranded plasmid DNA. Methods Enzymol. 153:3-11.

53. Warmington, J. R., R. B. Waring, C. S. Newlon, K. J. Indge, and S. G. Oliver. 1985. Nucleotide sequence characterization of Ty1-17, a class II transposon from yeast. Nucleic Acids Res. 13: 6679-6693.

54. Yanisch-Perron, C., J. Vieira, and J. Messing. 1985. Improved M13 phase cloning vectors and host strains: nucleotide sequences of the M13mp18 and pUC19 vectors. Gene 33:103-119. 